STATE OF ALASKA

DEPARTMENT OF NATURAL RESOURCES

Alaska Geologic Materials Center Data Report No. 386

\title{
No. 386
}

Talisman Energy Inc. 2011, Porosity, permeability, and grain density core analysis results from the FEX Limited Partnership Aklaq \#6 well and white light and ultra-violet photography of the FEX Limited Partnership Aklaq \#2, Aklaq \#6, and Aklaqyaak \#1 wells

Received April, 2011

All data reports may be downloaded free of charge from the DGGS website. 

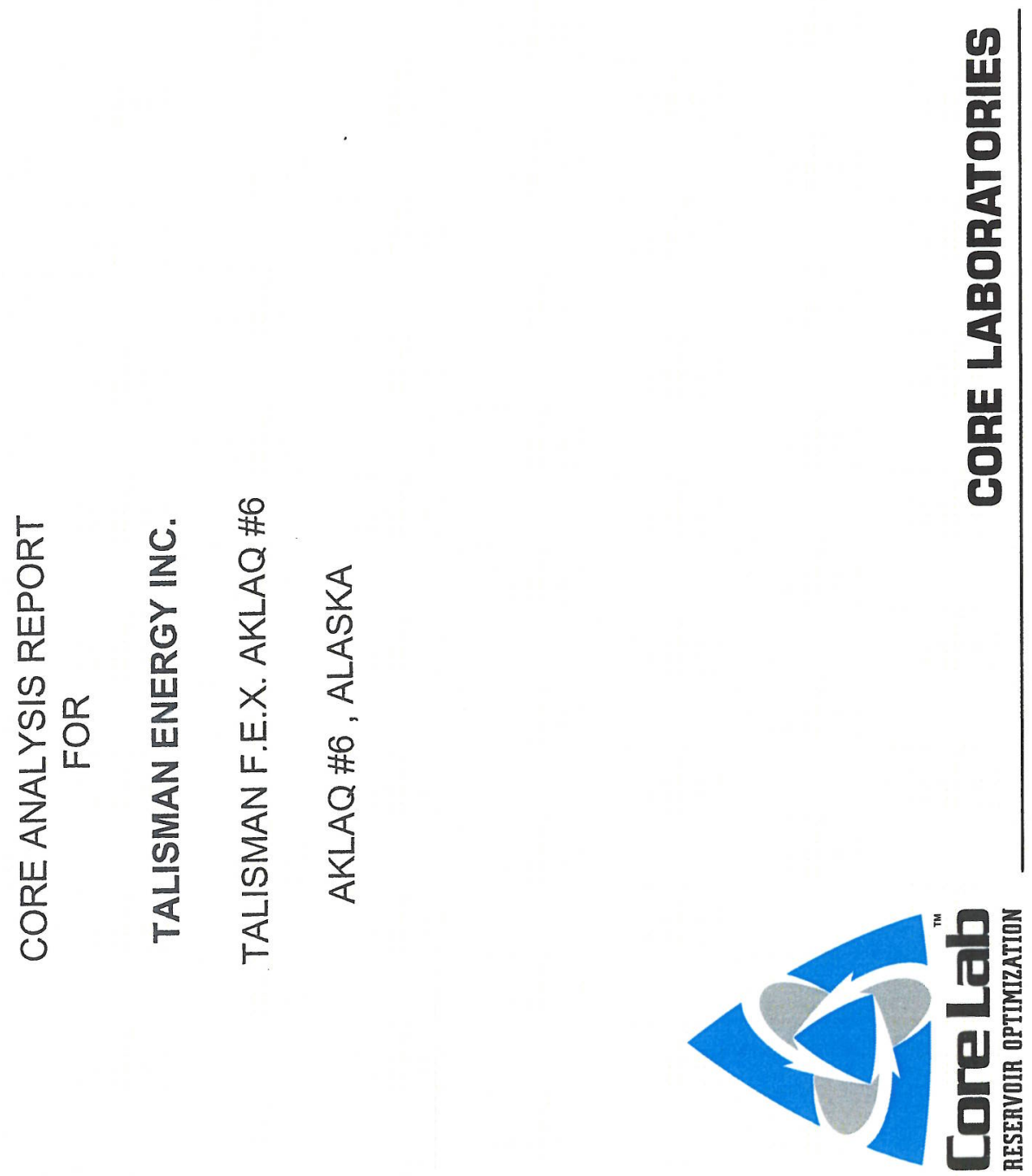

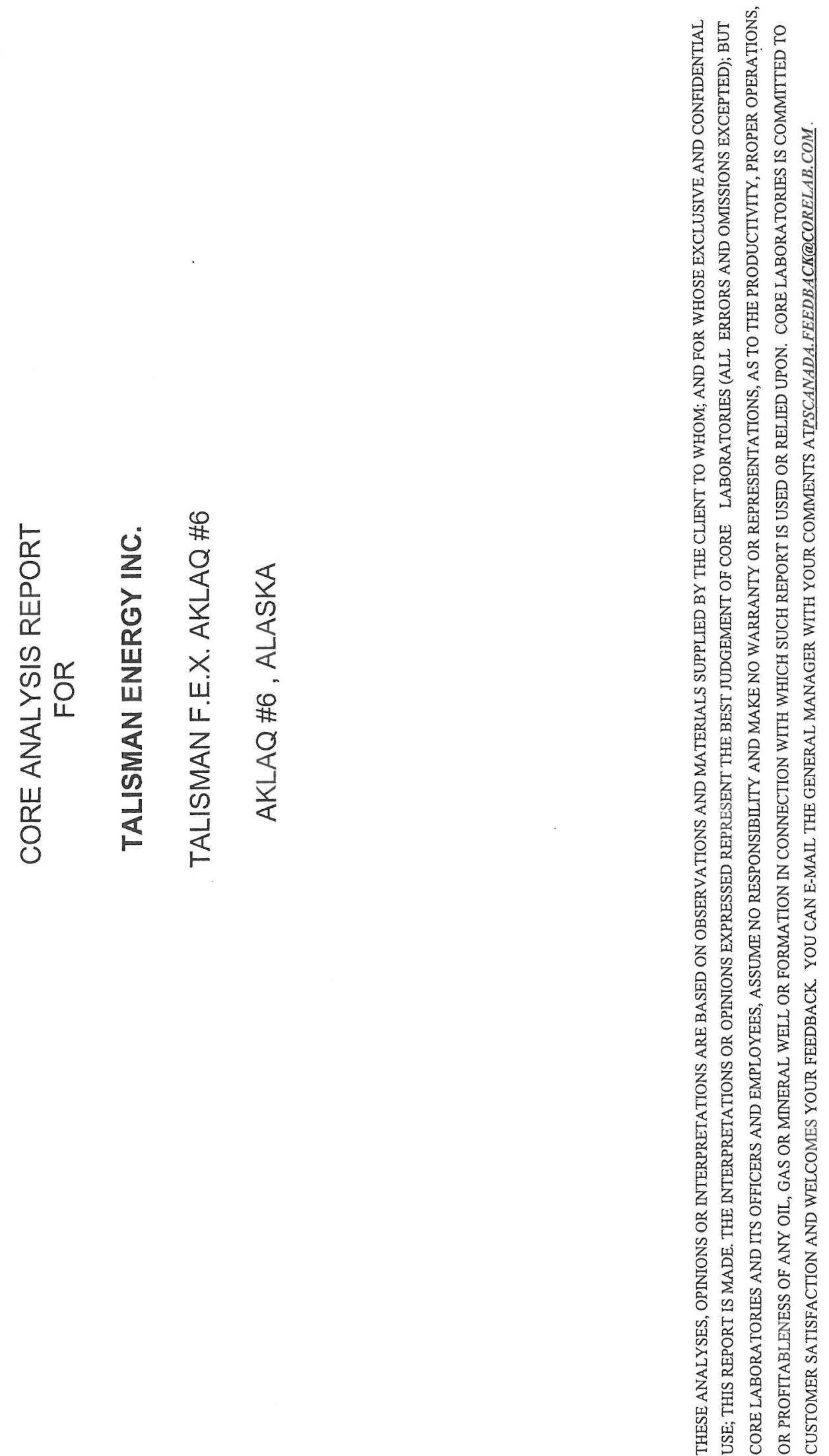
Talisman Energy Inc.

$3400,888-3^{\text {rd }}$ Street S.W.

Calgary, Alberta T2P 5C5

Attention: Mr. Jason Lavigne

Subject: TALISMAN F. E. X. AKLAQ \#6

Our File Number: 52131-07-0150

Diamond coring equipment and water base mud were used to core the subject well. The core was boxed at the wellsite and transported to our Calgary laboratory.

Prior to analysis, spectral gamma ray activity was measured.

1. Conventional, Plug Type Analysis (Humidity Dried)

Thirty-six samples (38.1 mm diameter) were drilled from the bulk core using water. The samples were dried in a humidity oven at $60^{\circ}$ Celsius and $50 \%$ relative humidity. Analysis includes porosity by Boyle's Law technique using helium as the gaseous medium and horizontal permeability to air.

The results of these analyses are reported in both tabular and graphical form.

Samples outside the $95 \%$ confidence interval (three standard deviations) on the Permeability X Porosity crossplot have been identified and have been checked and re-analyzed to ensure data integrity.

As a guide to evaluation and correlation with other data, statistics with tabulated cut-off ranges and plots are also presented herein.

The $1 / 3$ slabbed portion of the core material was photographed and six sets of white light and ultraviolet light, $216 \mathrm{~mm} \mathrm{X} 280 \mathrm{~mm}$ photographs and six compact disks will be forwarded to your office under a separate cover.

Thank you for the opportunity to be of service.

Yours truly,

CORE LABORATORIES CANADA, LTD.

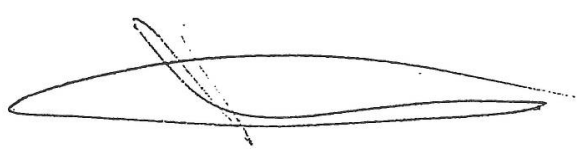

David J. Brooks

Supervisor, Routine Rock Properties

$\mathrm{DJB} / \mathrm{wz}$

enclosures

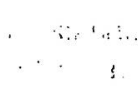




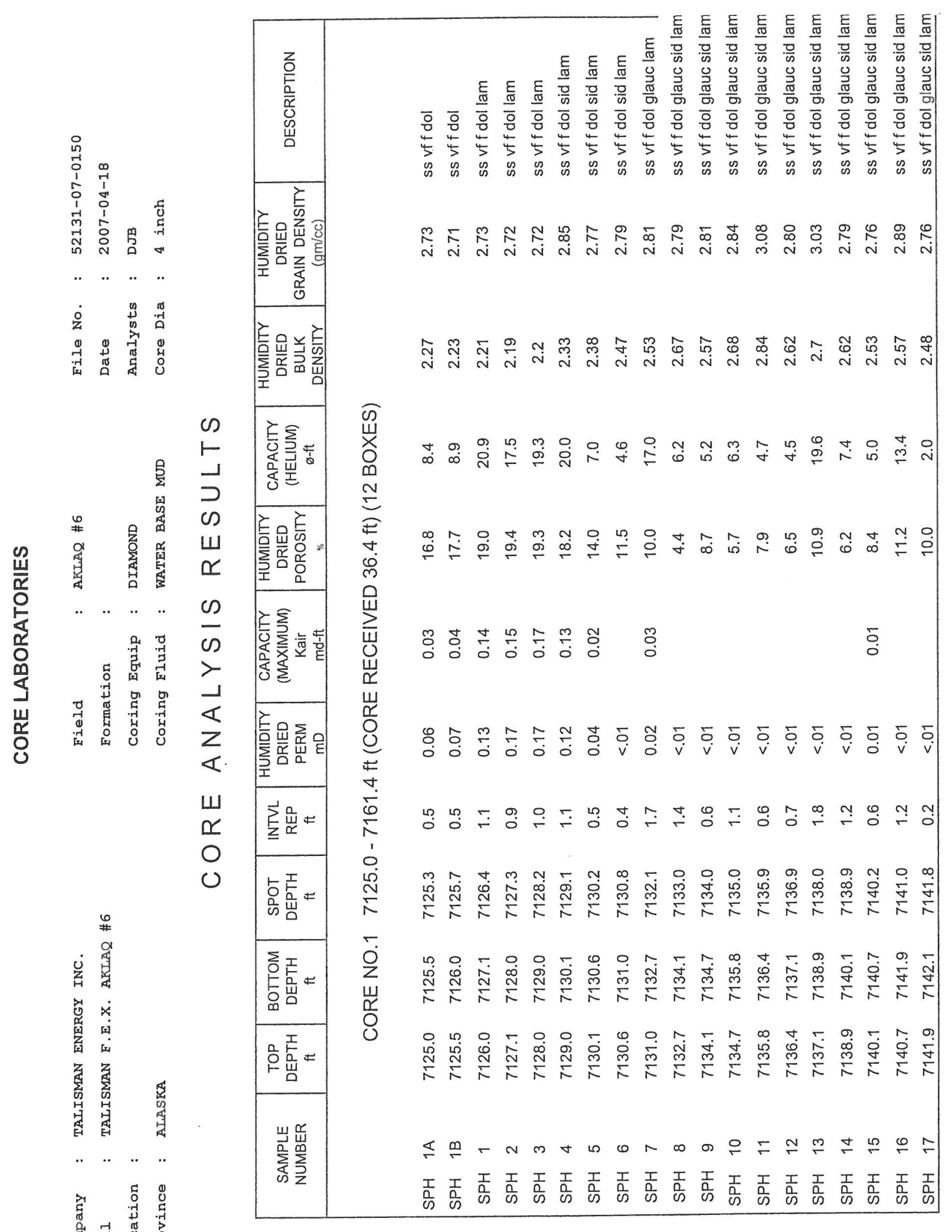

$\frac{1}{5}$
$\frac{1}{0}$
0
0
0 


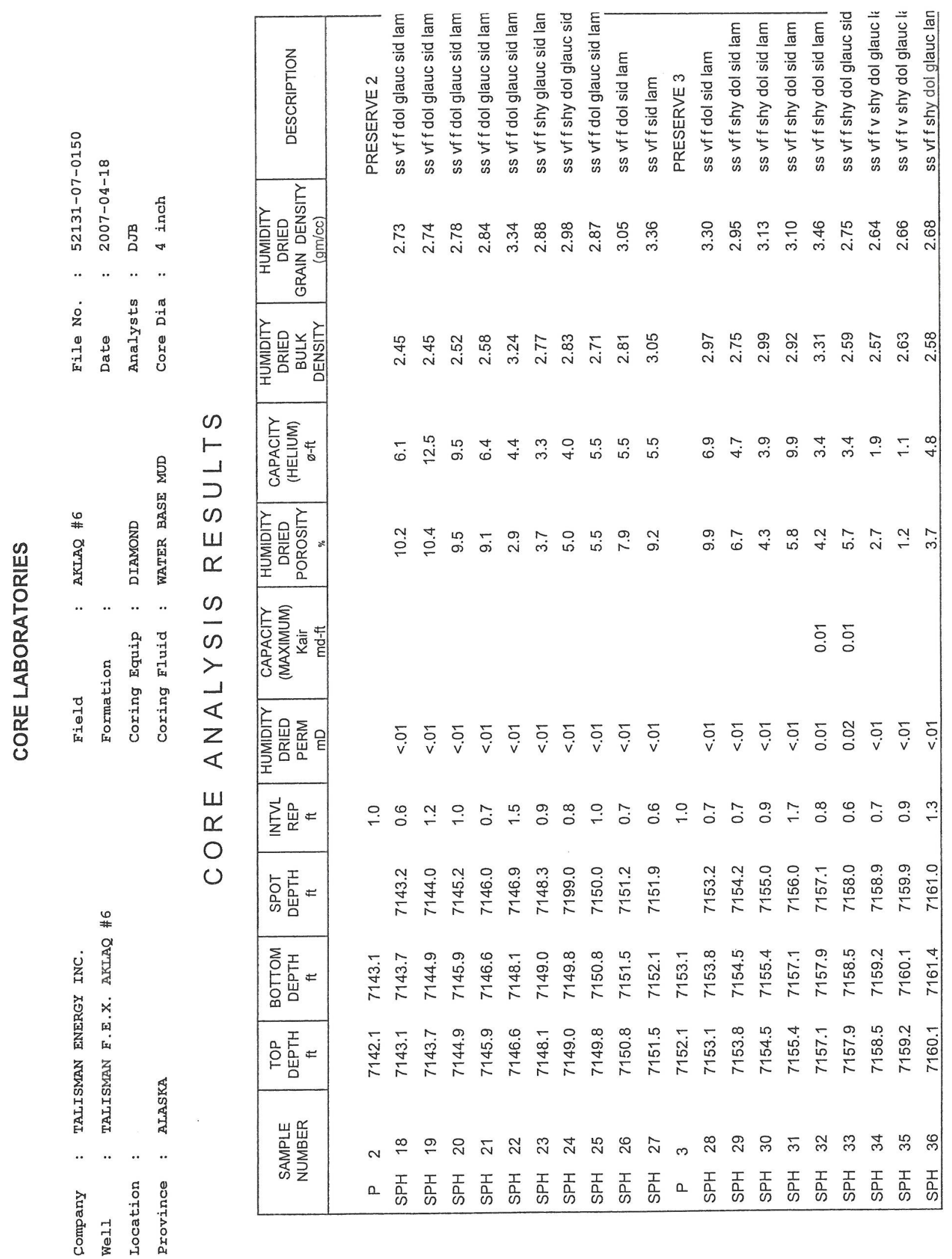



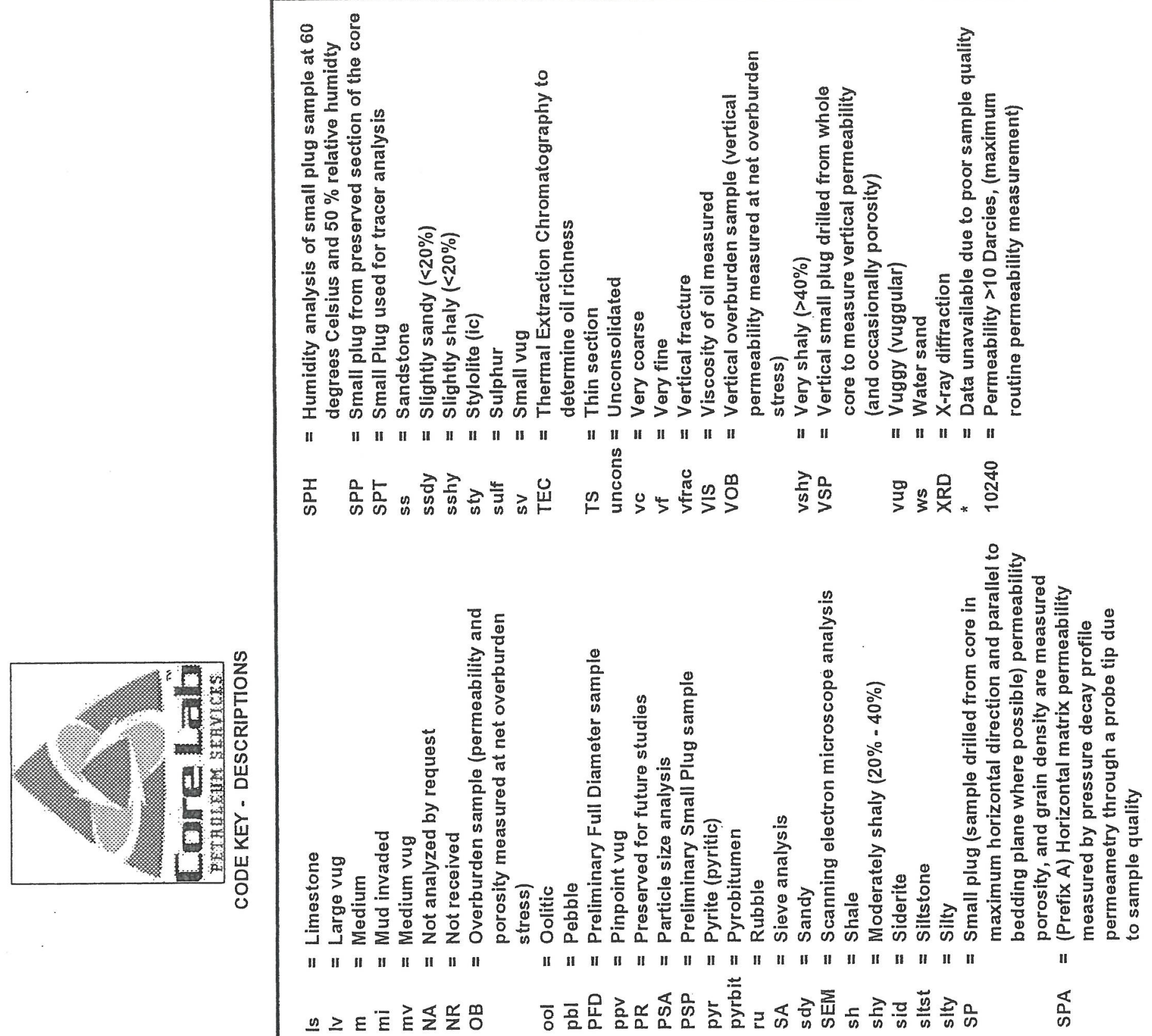

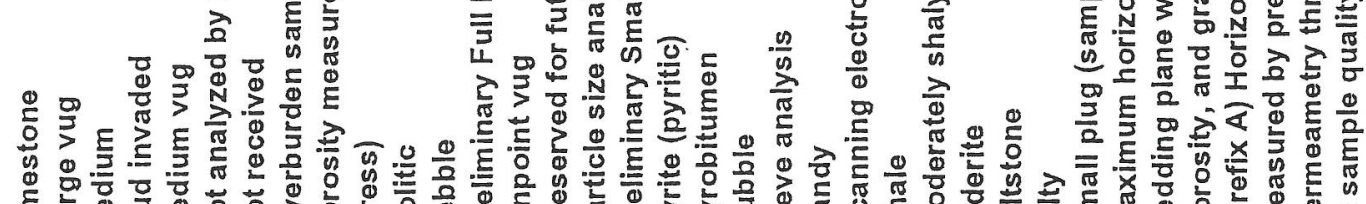

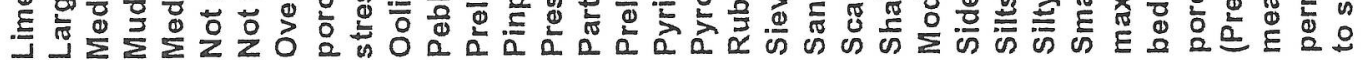

"

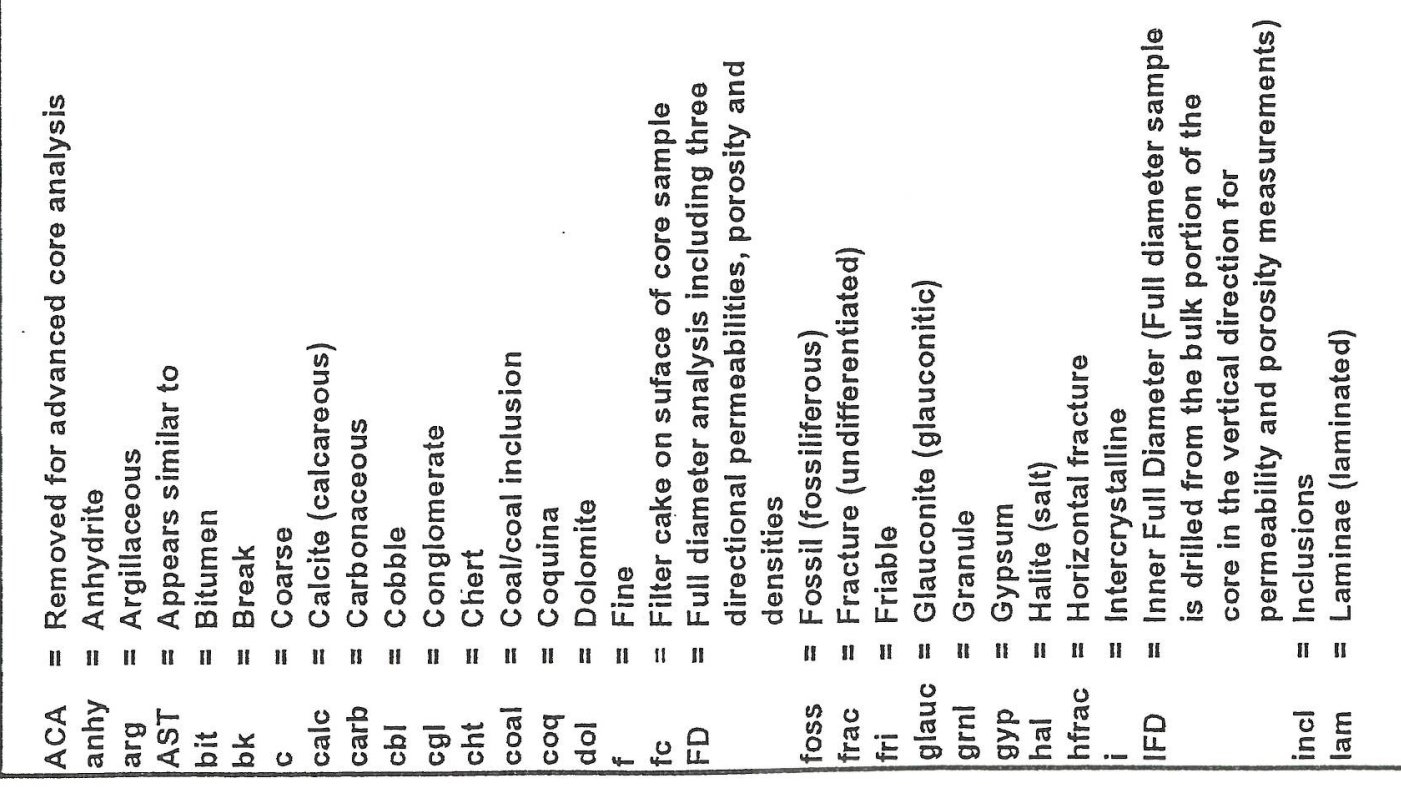




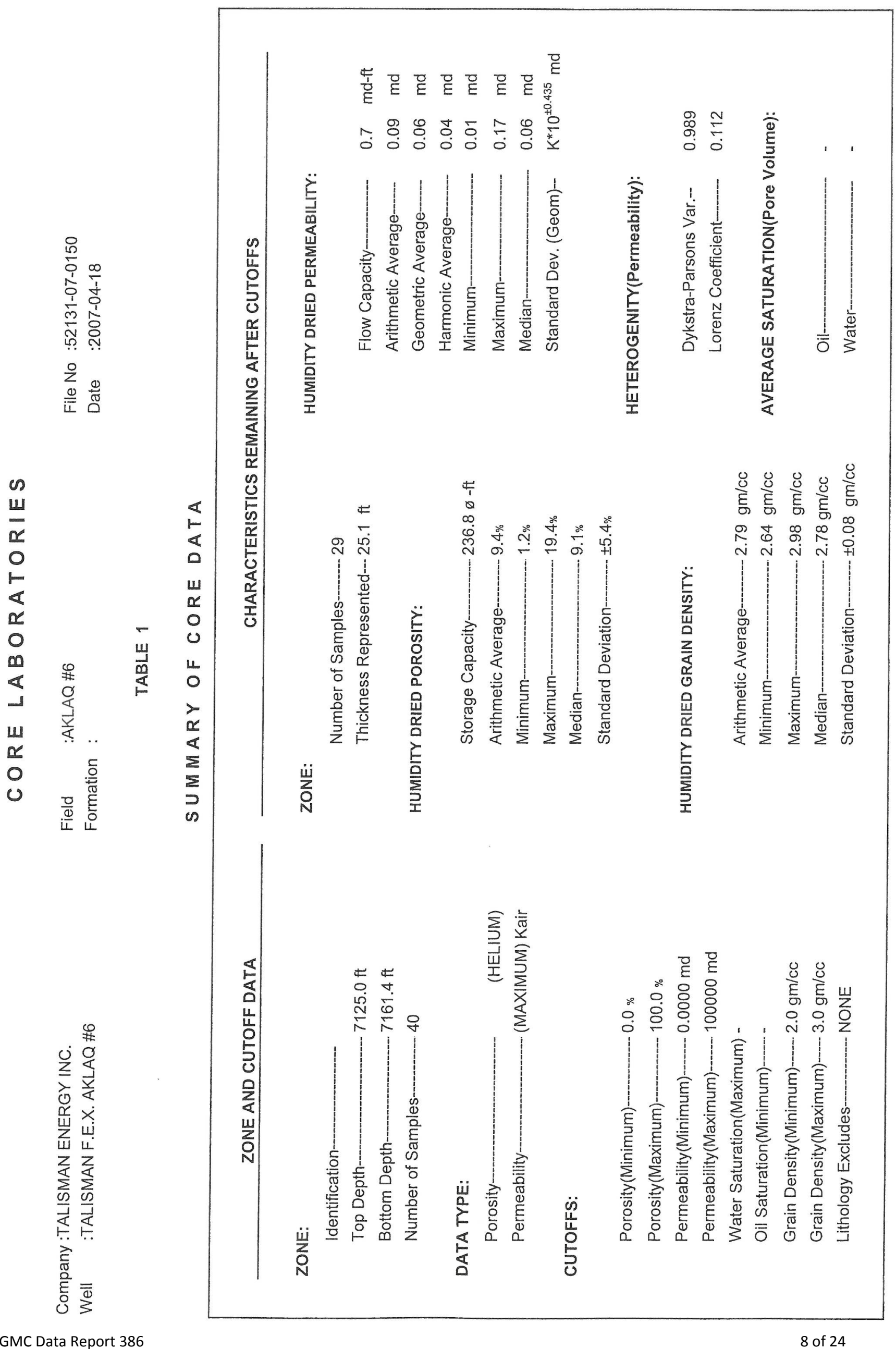




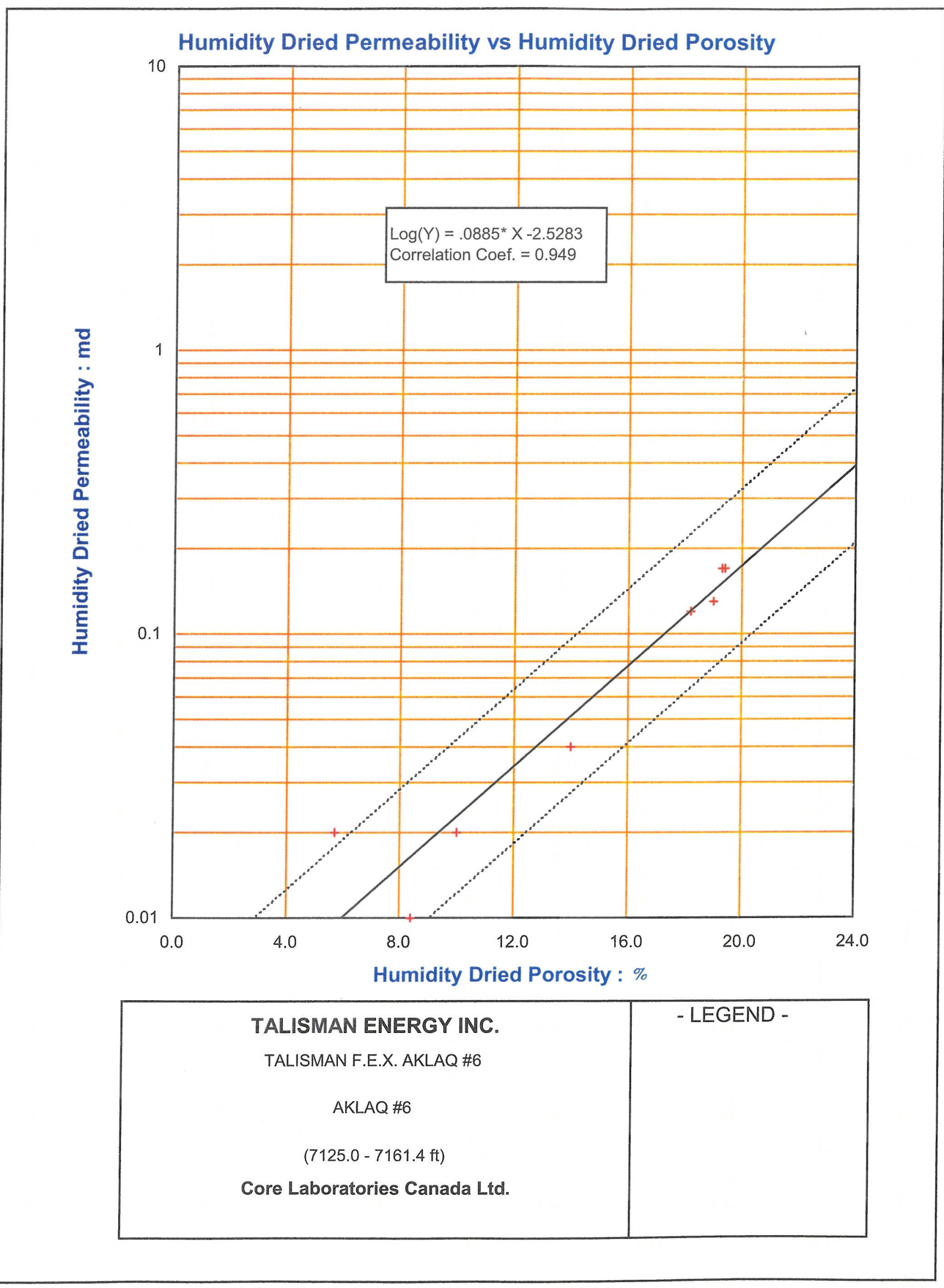




\title{
CORE SPECTRAL GAMMA
}

\author{
TALISMAN ENERGY INC. \\ TALISMAN F。 E. X.AKLAQ \#6 \\ $(7125.0$ - $7161.4 \mathrm{ft})$ \\ 5 inches - 100 feet \\ Core Laboratories Canada Ltd.
}

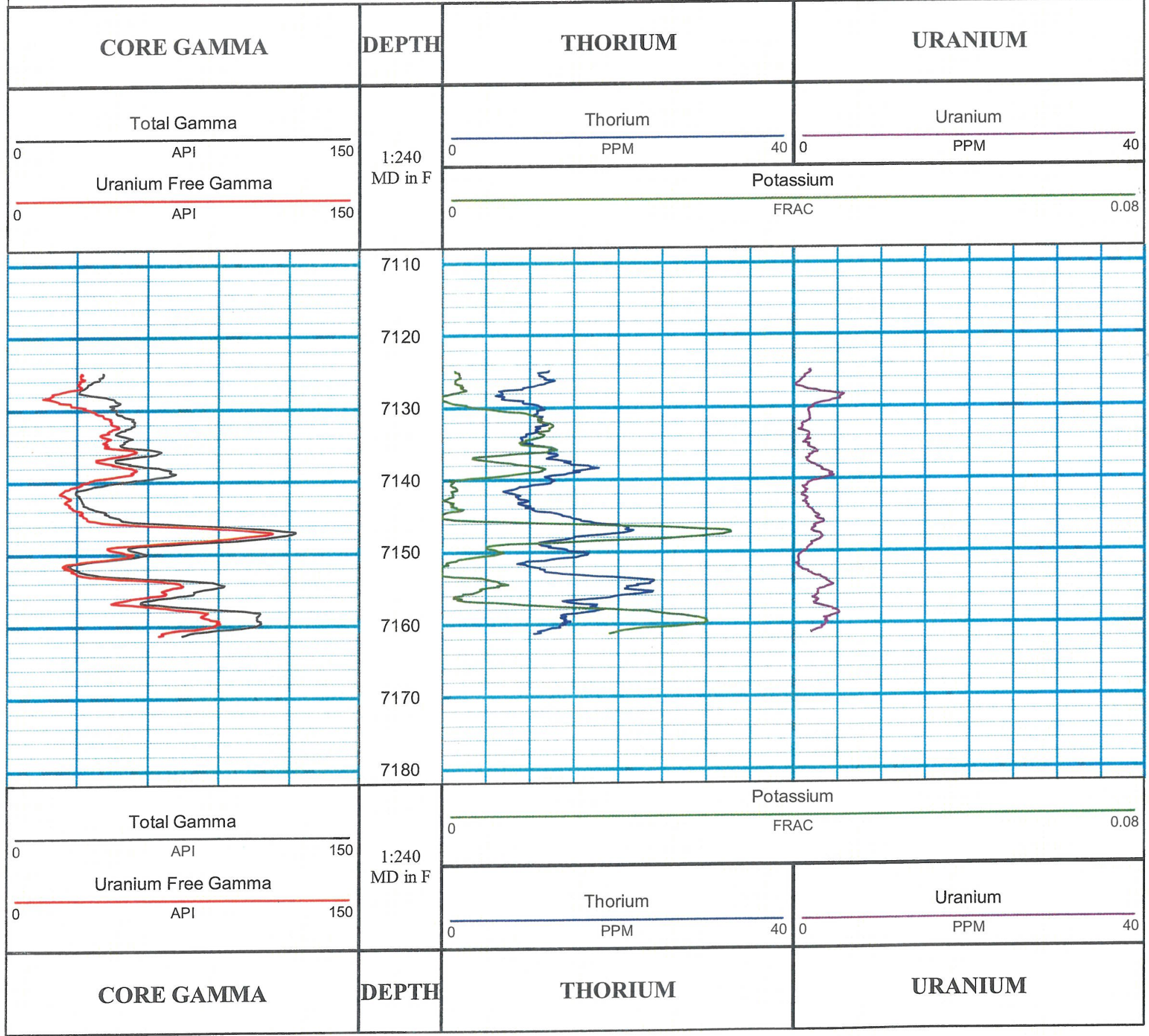




\section{CORRELATION COREGRAPH}

\section{TALISMAN ENERGY INC. \\ TALISMAN F。 E. X. AKLAQ \#6 \\ $(7125.0$ - $7161.4 \mathrm{ft})$}

5 inches - 100 feet

Core Laboratories Canada Ltd.

\section{Lithology}

Limestone

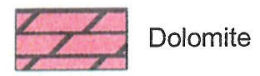

$\because \because \because$ Sandstone

$0 .-1$ Conglomerate

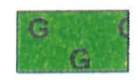

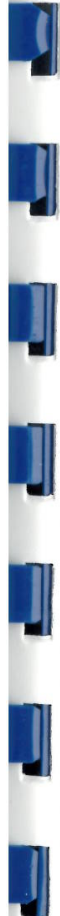

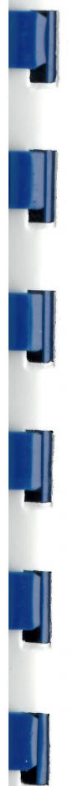

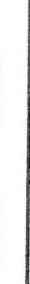

Total Gamma

GAMMA RAY (API) API

Uranium Free Gamma

API

150

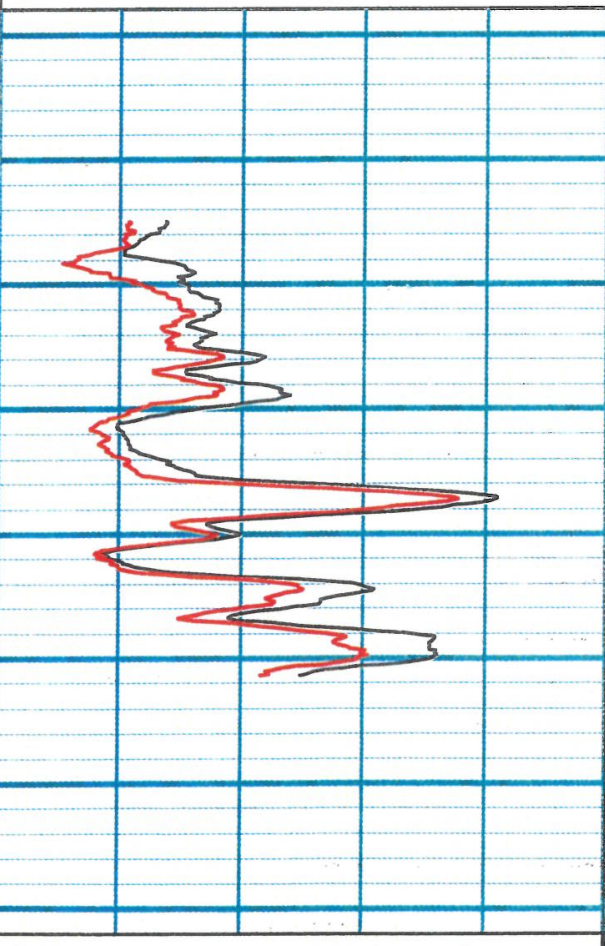

Total Gamma

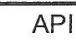

API

GAMMA RAY (API)
Uranium Free Gamma

$$
7140
$$

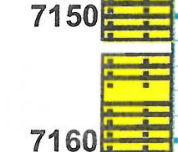

$\mathrm{MD}$ in $\mathrm{F}$

DEPTH $\because \because \because$

Glauconite

DEPTH

1:240

$\mathrm{MD}$ in $\mathrm{F}$

50.0

HUMWITY DRIED POROSITY /HUMIDITY DRIED GRAIN DENSITY

2

7110

7120

7130

$=$

7150

Shaley Sandstone

$\longrightarrow$ Siltstone

Coal
Helium Porosity

Core Grain Density

GM/CC

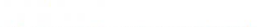

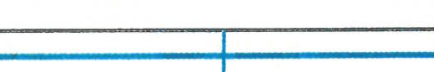

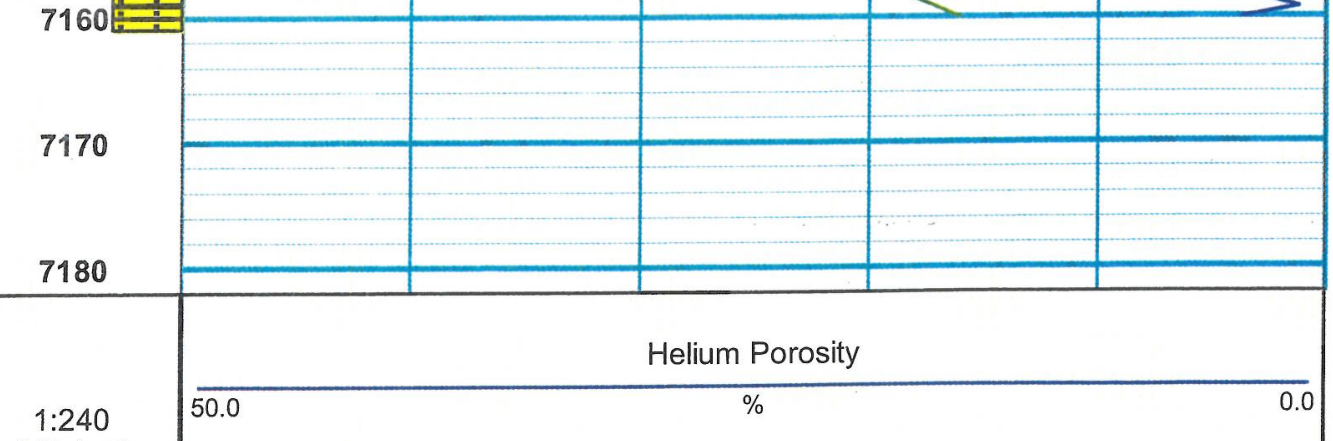

Core Grain Density

$\mathrm{GM} / \mathrm{CC}$

HUMIDITY DRIED POROSITY /HUMIDITY DRIED GRAIN DENSITY 


\section{CORRELATION COREGRAPH}

TALISMAN ENERGY INC.

TALISMAN F。 E. X. AKLAQ \#6

(7125.0 - $7161.4 \mathrm{ft})$

5 inches - 100 feet

Core Laboratories Canada Ltd.

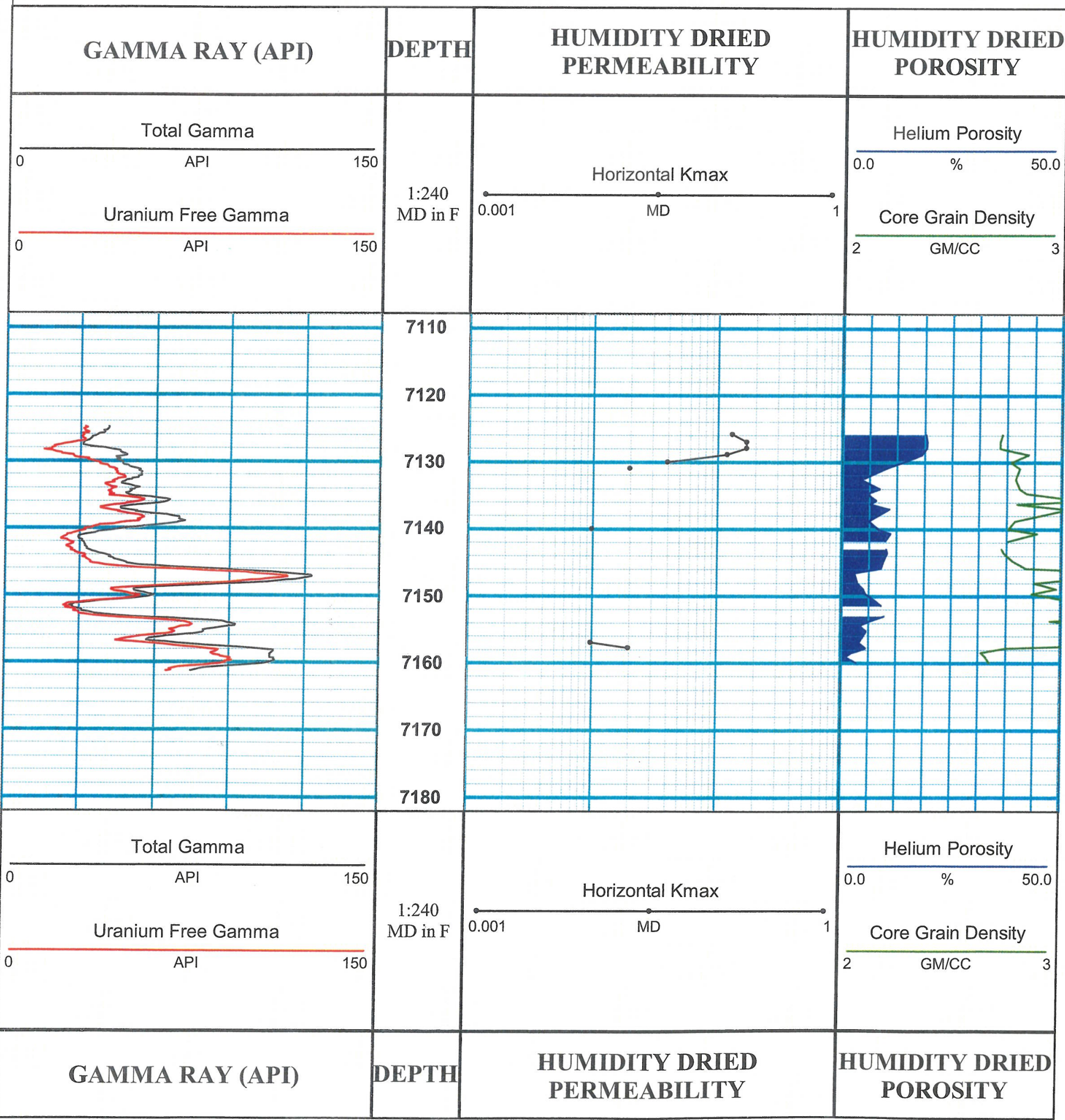


TALISMAN ENERGY INC.

TALISMAN F.E.X. AKLAQ \#6

\section{Core \#1}

Top $7125.0 \mathrm{ft}$

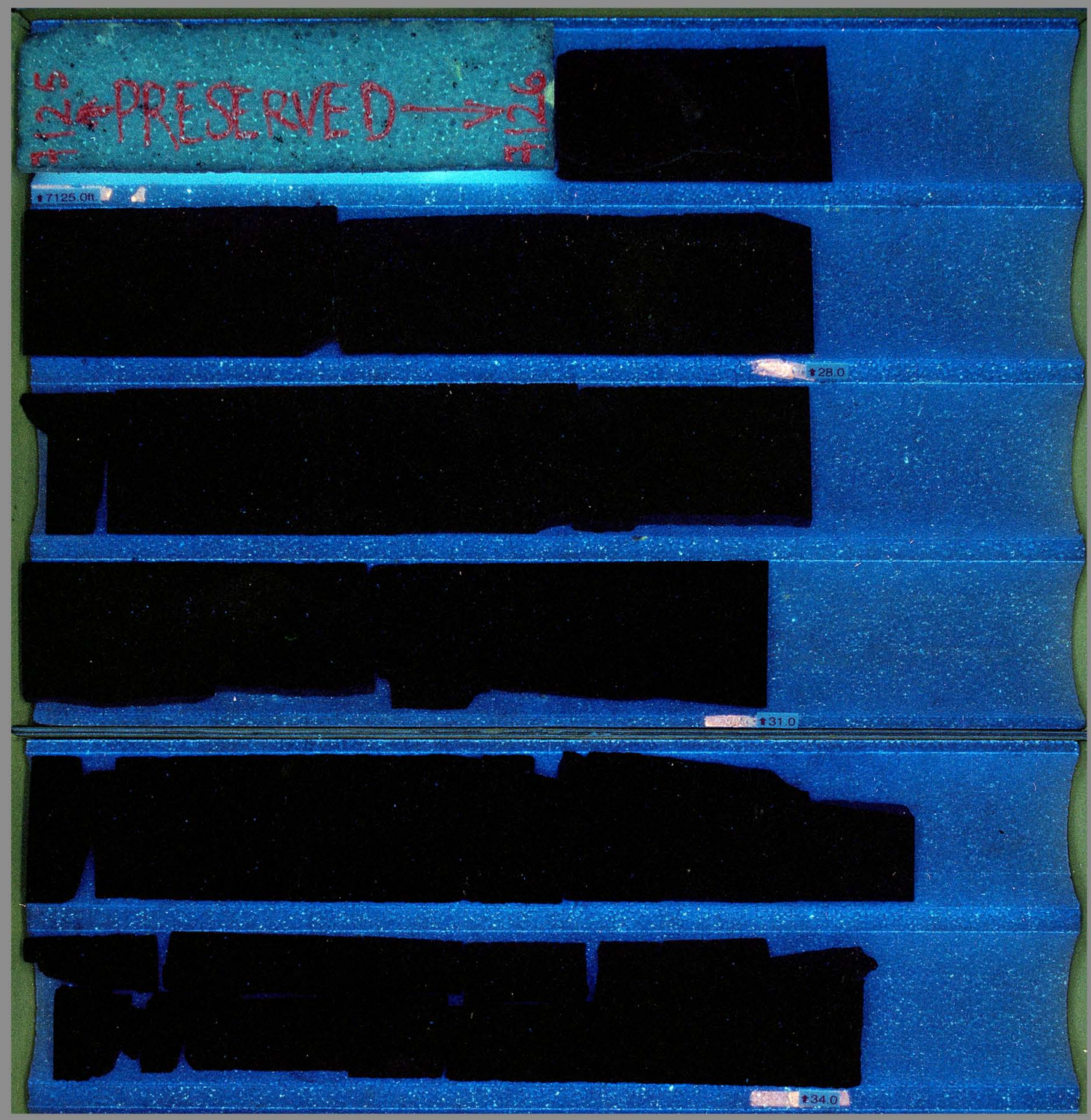

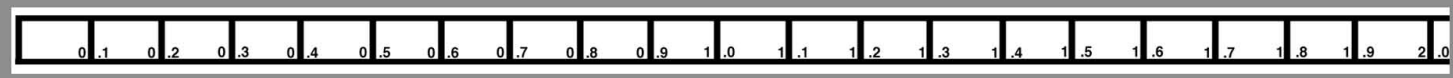
Bottom 7134.1 ft

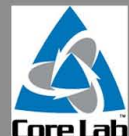

GMC Data Report 386

\section{Care Lab \\ 它}

10

TA L I 1550 M A

KODAK Color Control Patehes

$E N E R \quad G$ 


\section{Core \#1}

Top $7134.1 \mathrm{ft}$
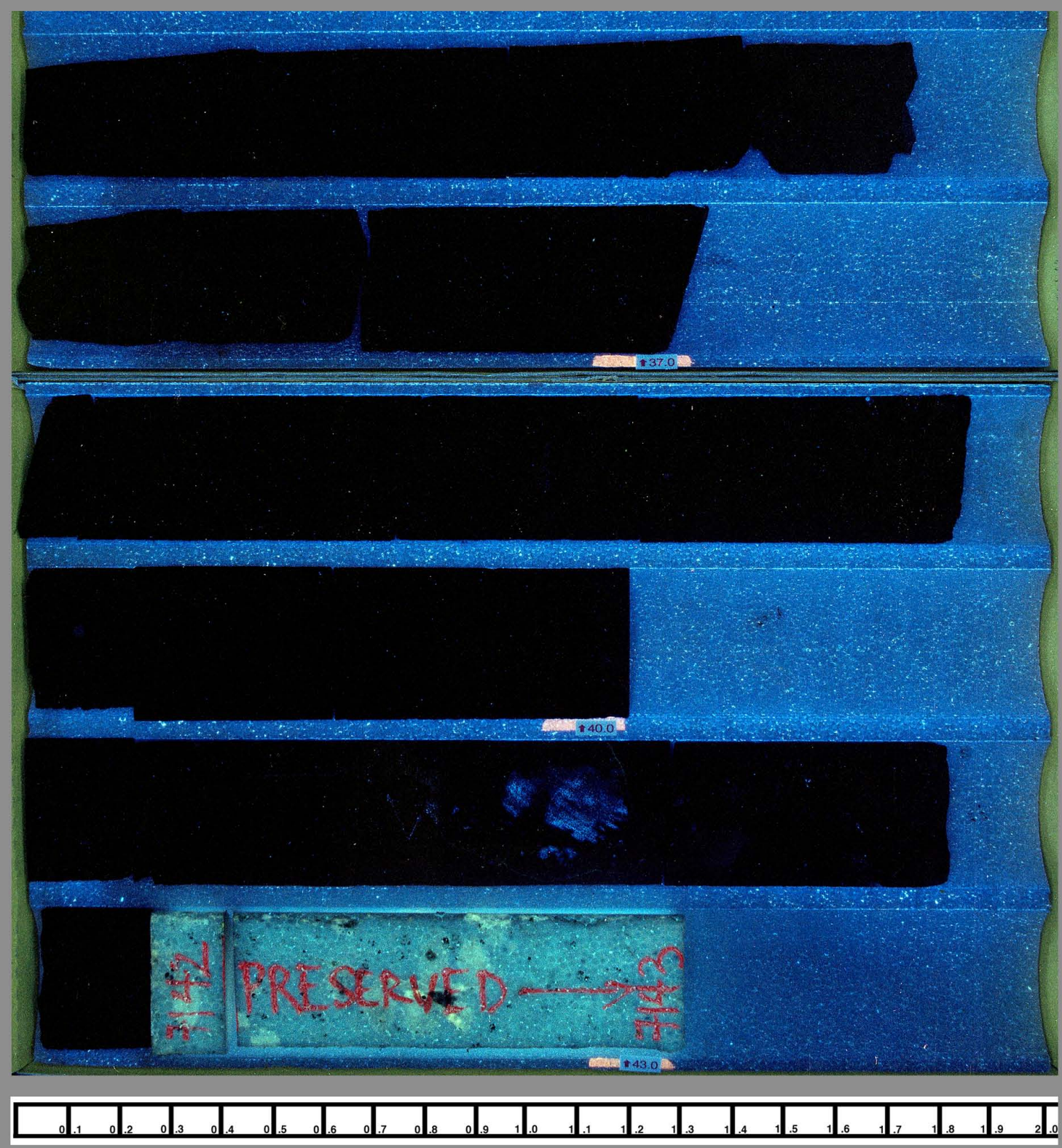

Bottom $7143.1 \mathrm{ft}$

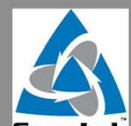

GMC Data Report 386

\section{Care Lab}

GMC Data Report 386
TALI田 $\mathbf{A}$ 


\section{Core \#1}

Top $7143.1 \mathrm{ft}$

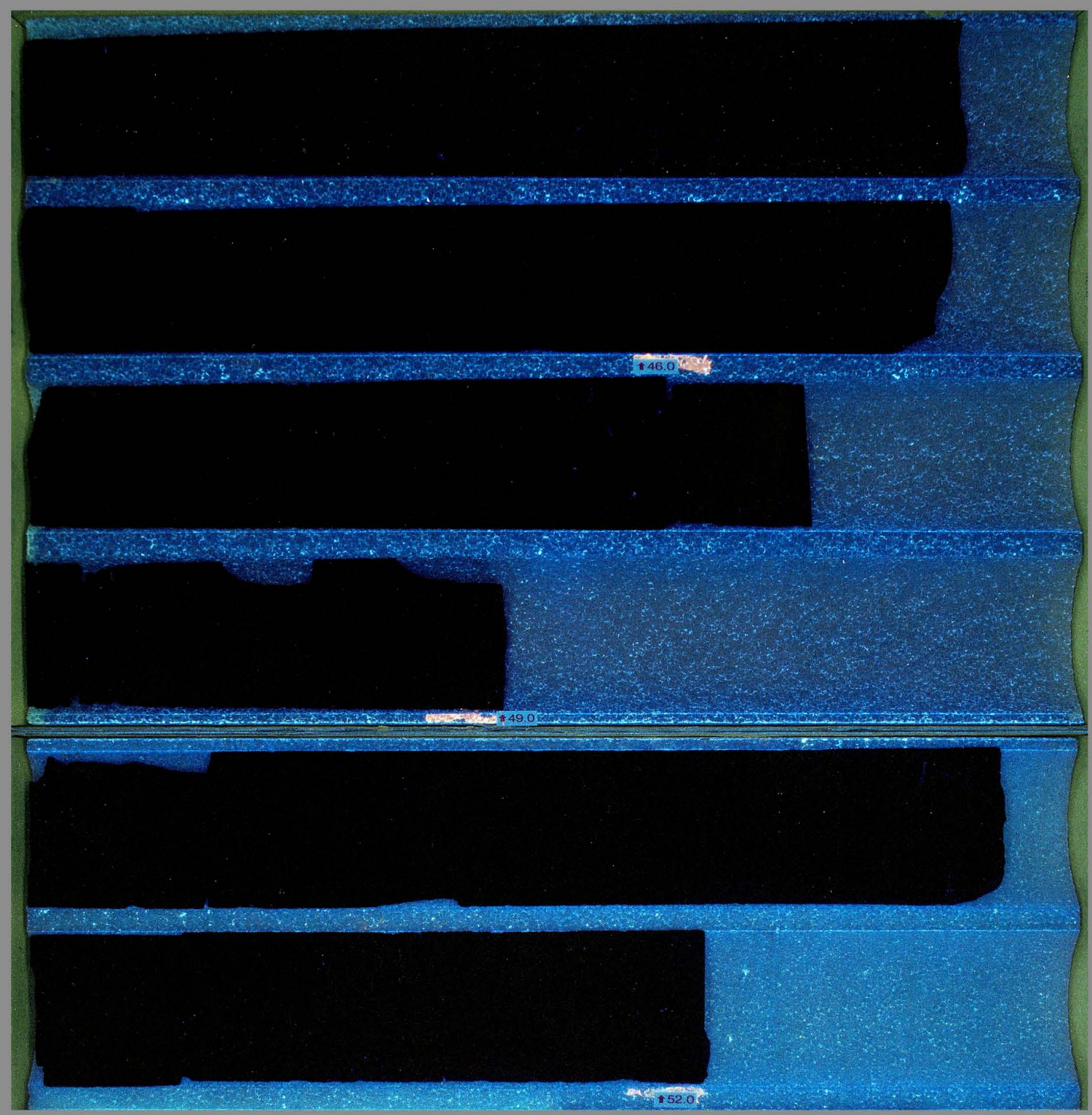

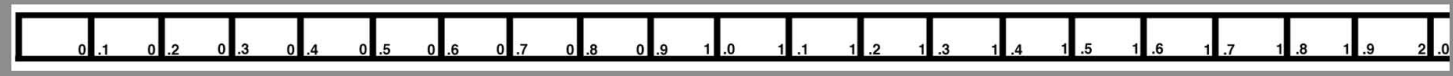

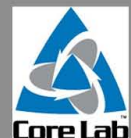

GMC Data Report 386

\section{Care Lab}

16

TA L I 150 $\mathbf{A M} \mathbf{N}$ 


\section{TALISMAN F.E.X. AKLAQ \#6}

\section{Core \#1}

\section{Top $7152.1 \mathrm{ft}$}

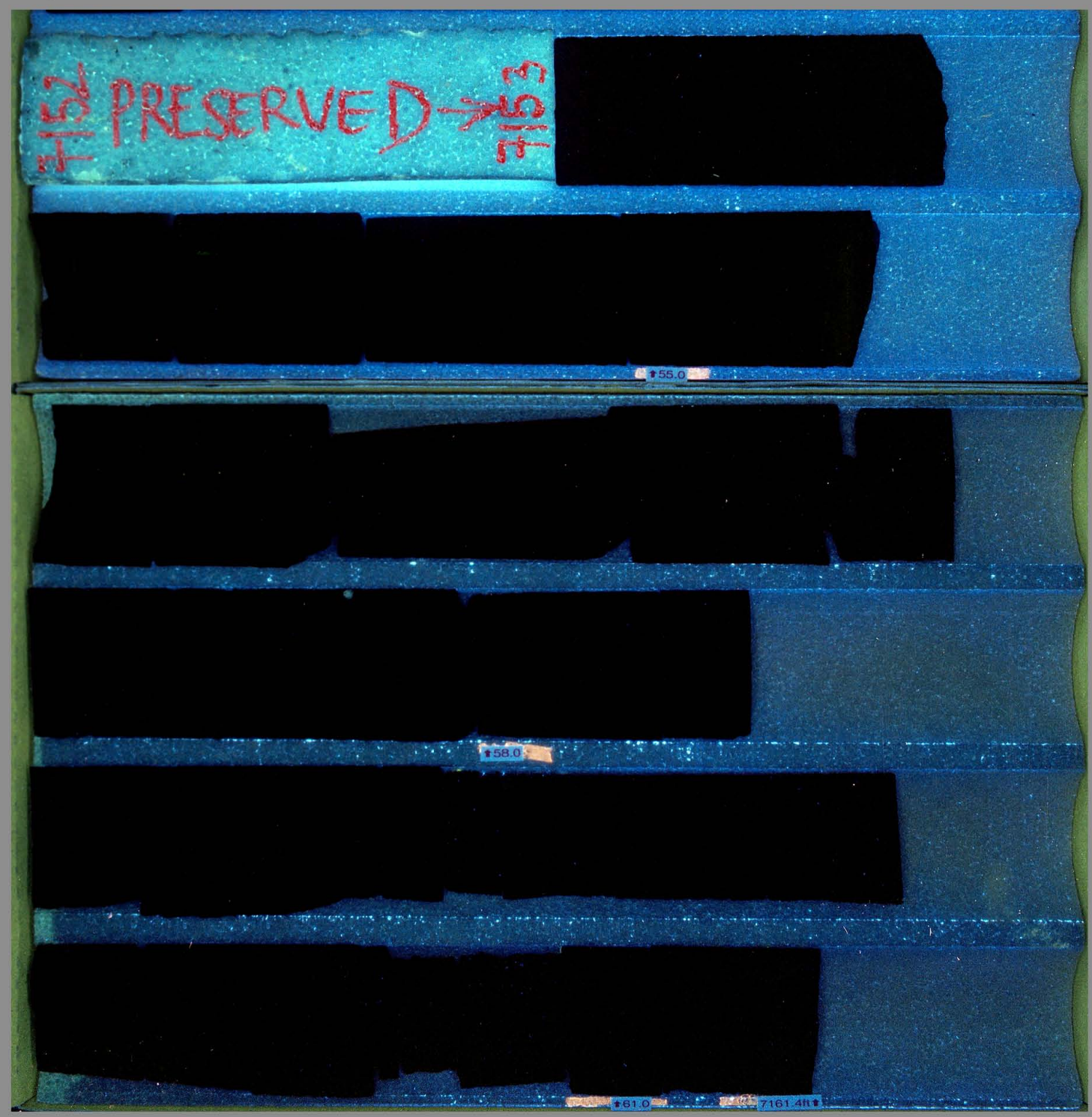

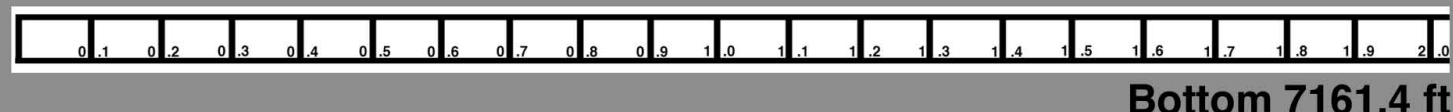

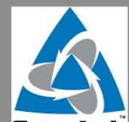

GMC Data Report 386

\section{Core Lab}

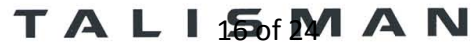

E $N$ E $R \quad G$


TALISMAN ENERGY INC.

TALISMAN F.E.X. AKLAQ \#6

\section{Core \#1}

Top $7125.0 \mathrm{ft}$
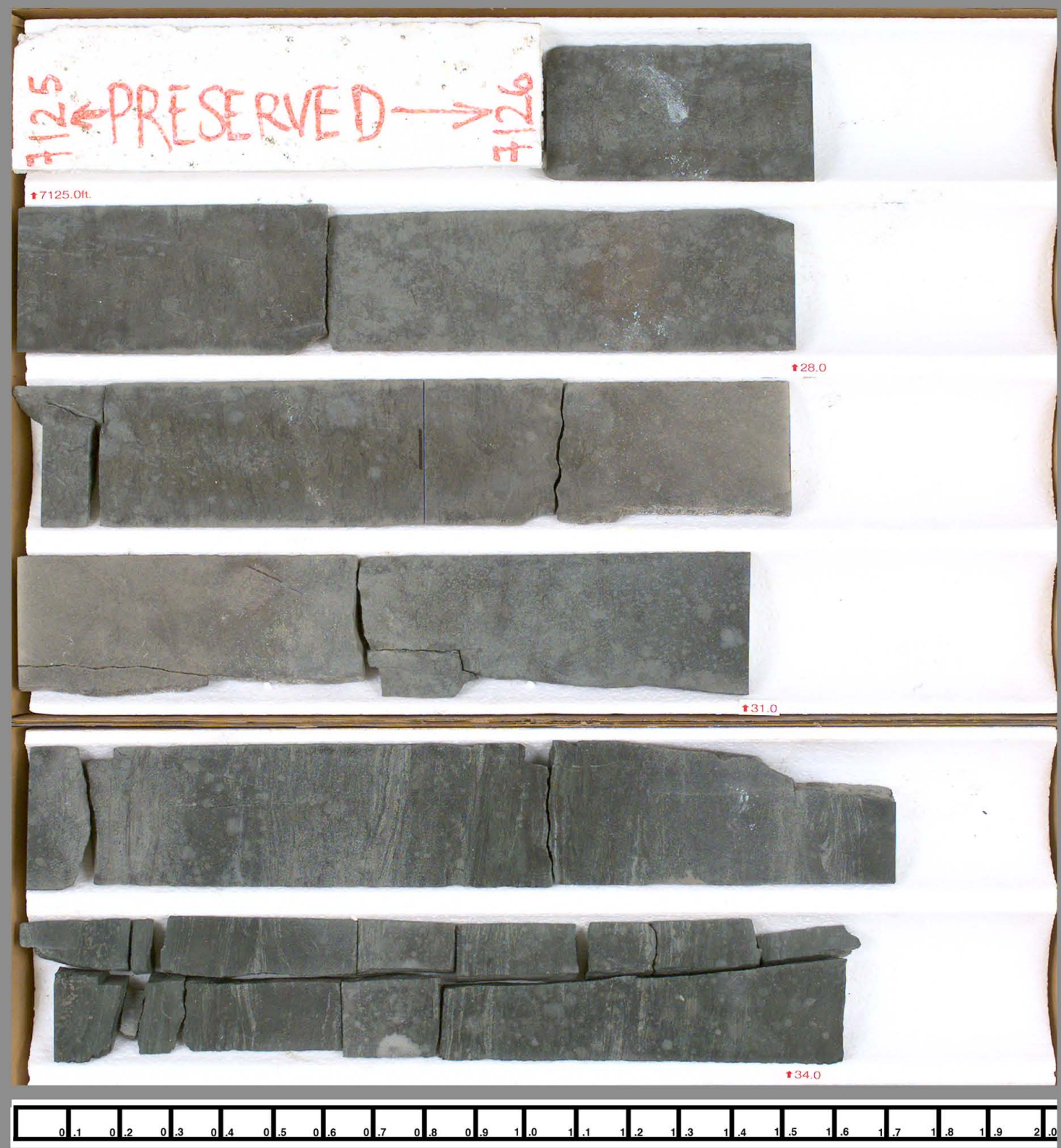
Bottom $7134.1 \mathrm{ft}$

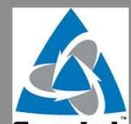

Core Lab

GMC Data Report 386

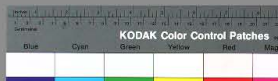

단

TA L I 1 
TALISMAN ENERGY INC.

TALISMAN F.E.X. AKLAQ \#6

\section{Core \#1}

Top $7134.1 \mathrm{ft}$
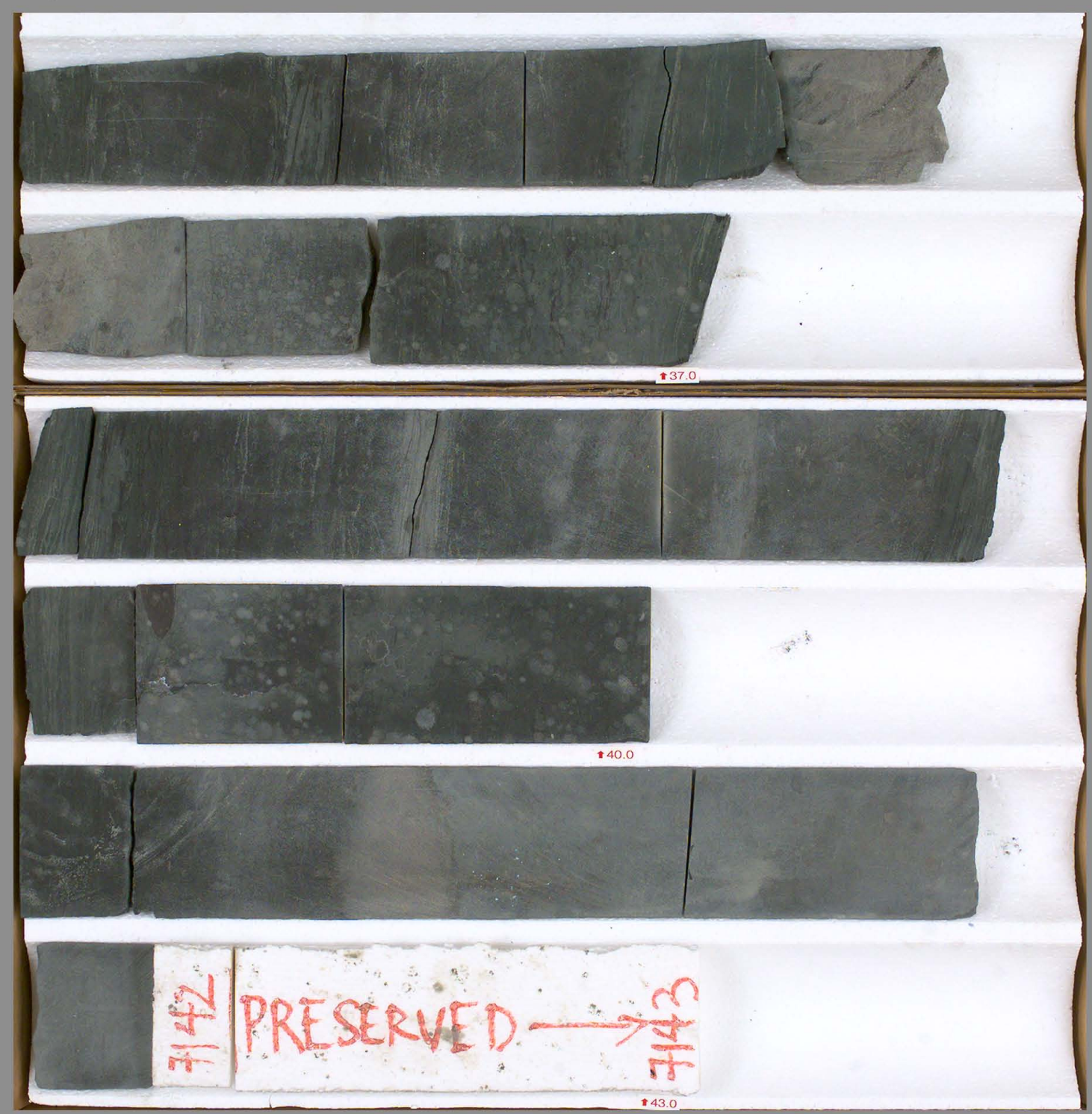

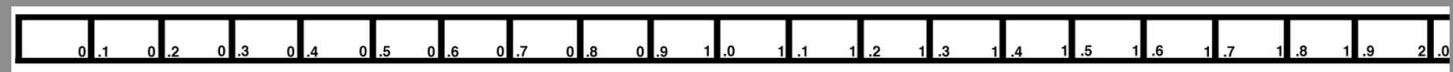
Bottom $7143.1 \mathrm{ft}$

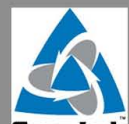

Core Lab

GMC Data Report 386

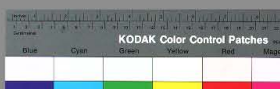

T A L I 1500 A N 6 


\section{Core \#1}

Top $7143.1 \mathrm{ft}$
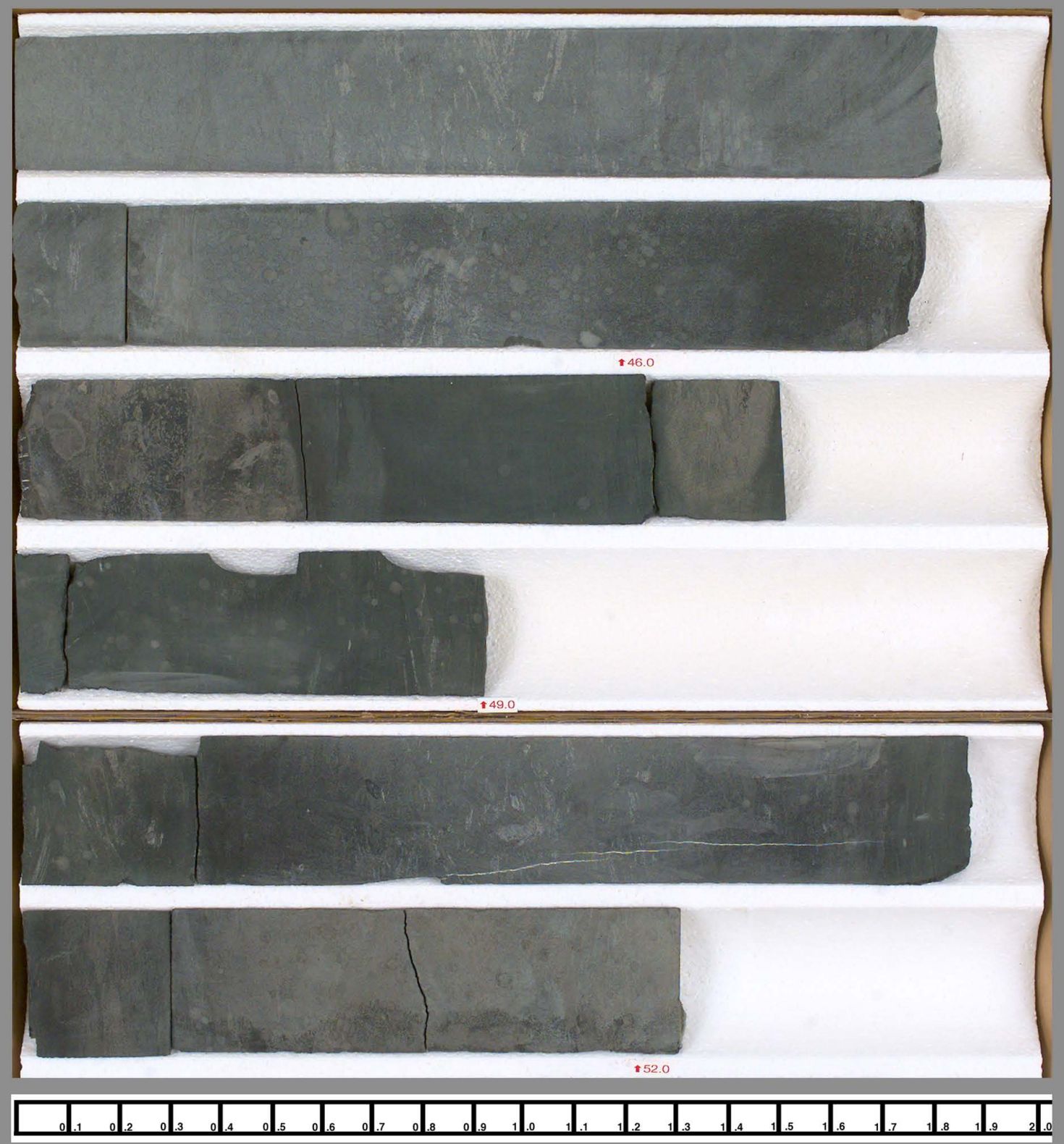
Bottom $7152.1 \mathrm{ft}$

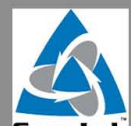

\section{Core Lab}

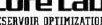

KODAK color Control Patehes

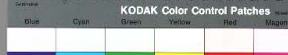

(6)

TA L I 19of A N 
TALISMAN ENERGY INC.

TALISMAN F.E.X. AKLAQ \#6

\section{Core \#1}

Top $7152.1 \mathrm{ft}$
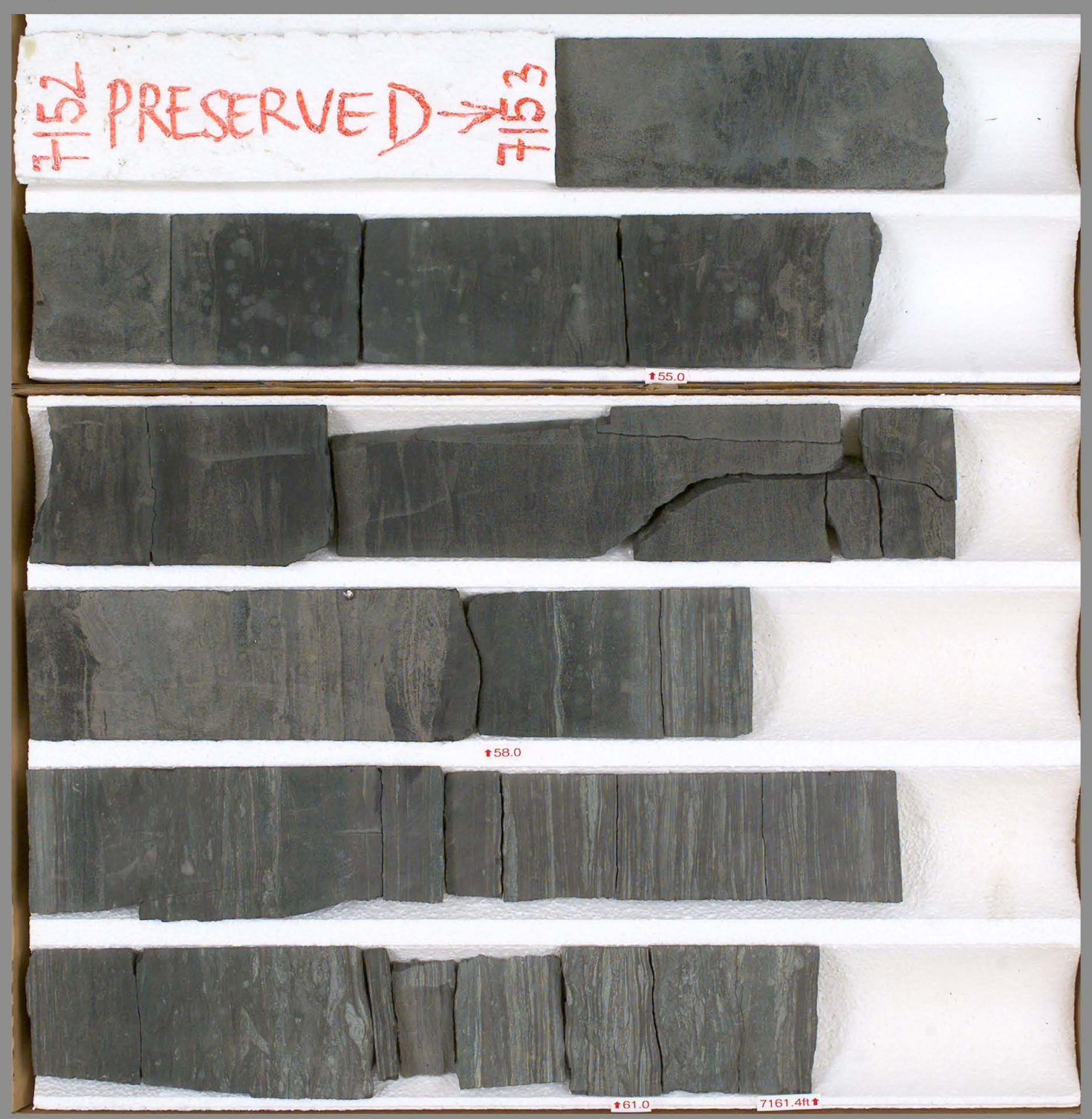

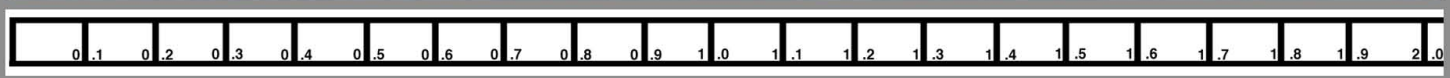
Bottom $7161.4 \mathrm{ft}$

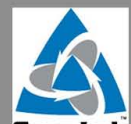

\section{Care Lab}

GMC Data Report 386

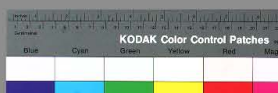

TA L I 250 A N 6 


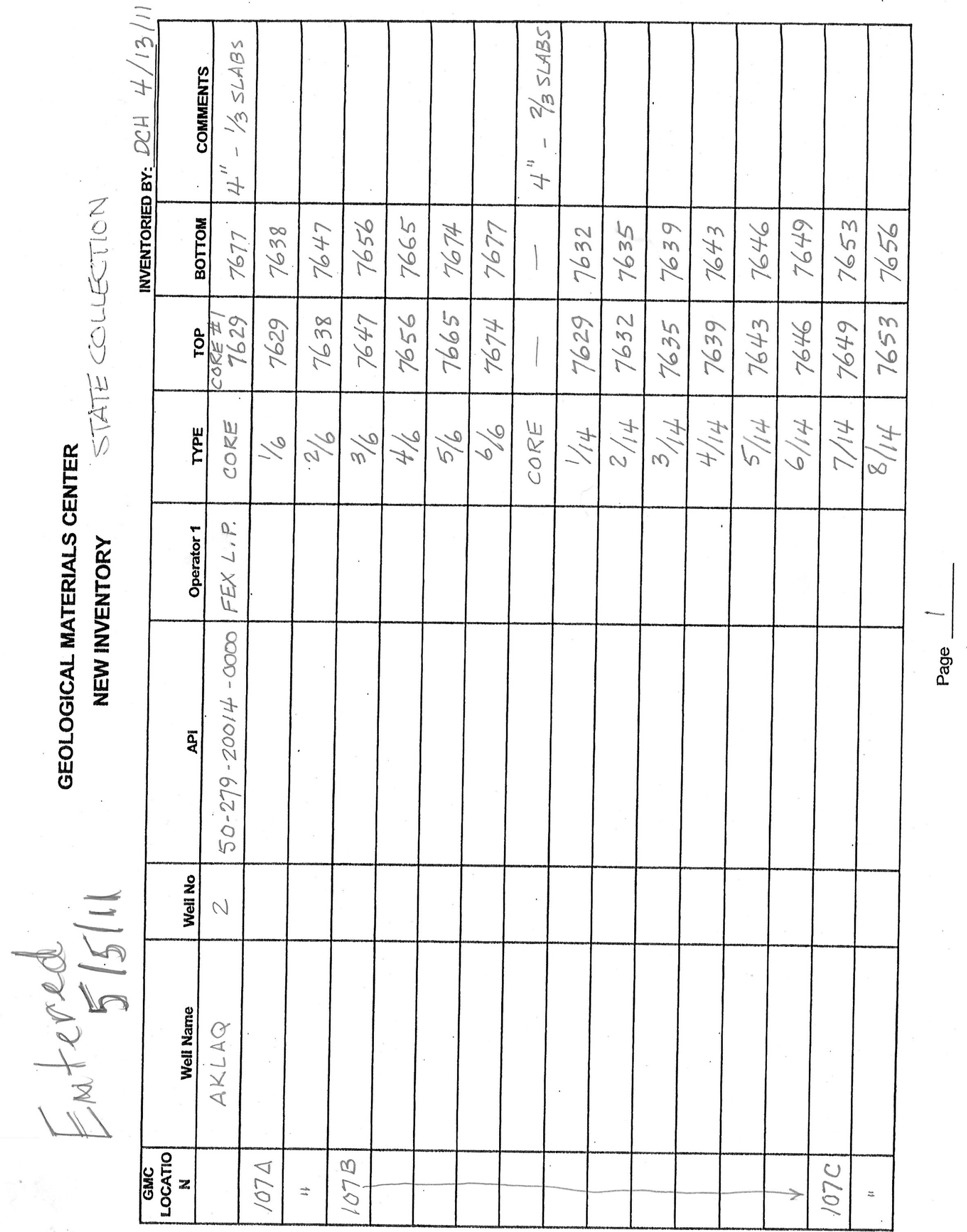




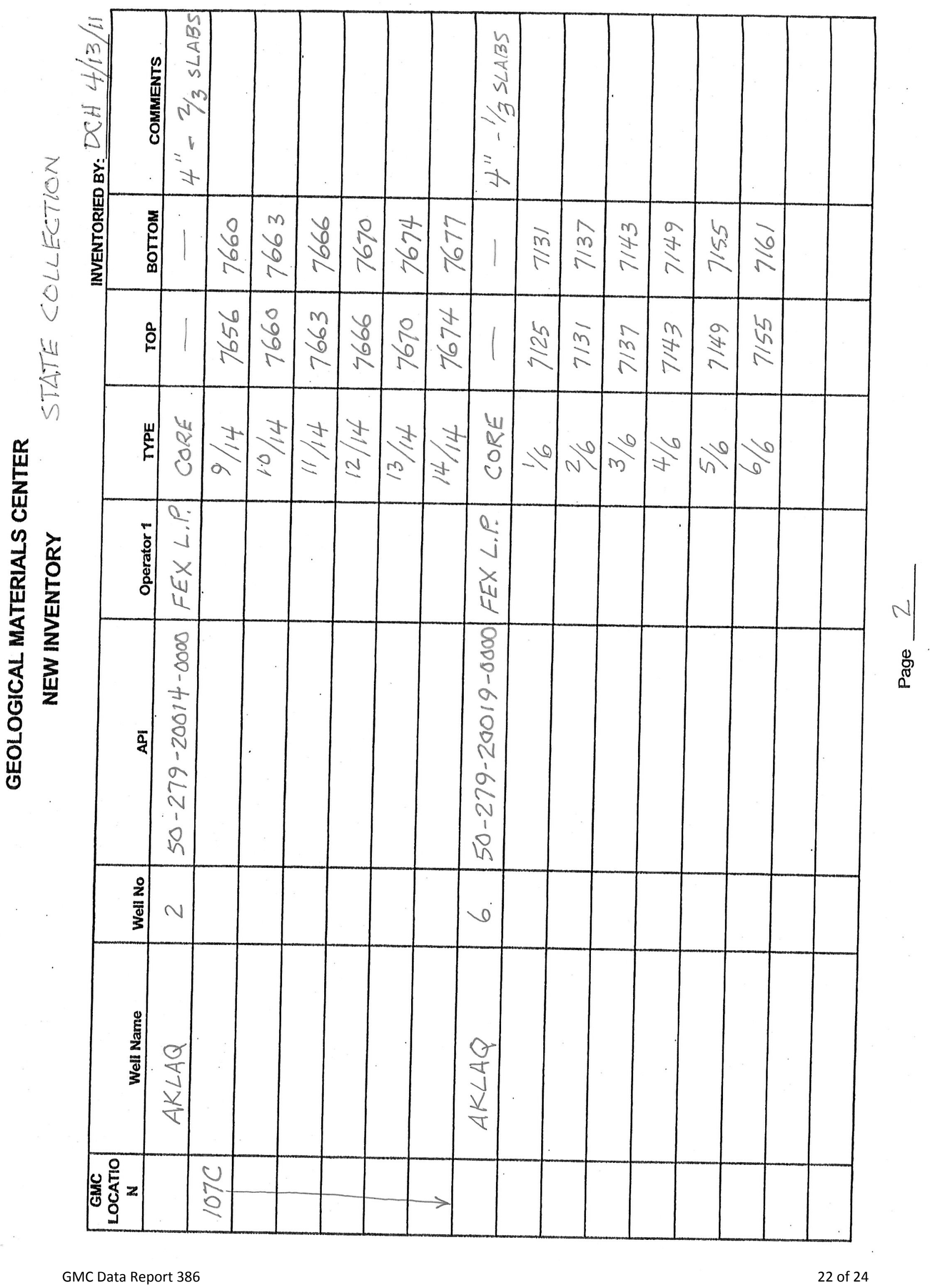




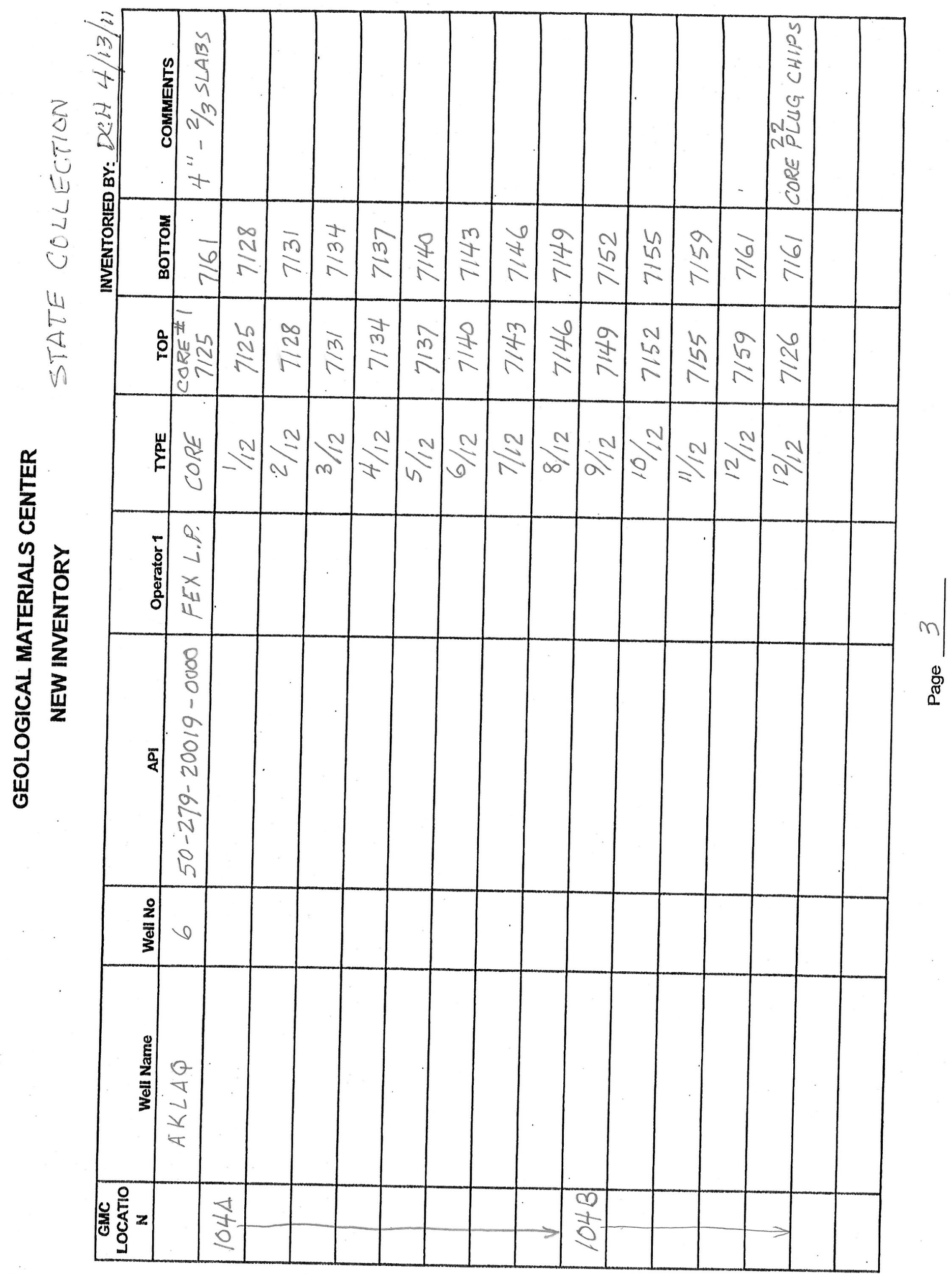




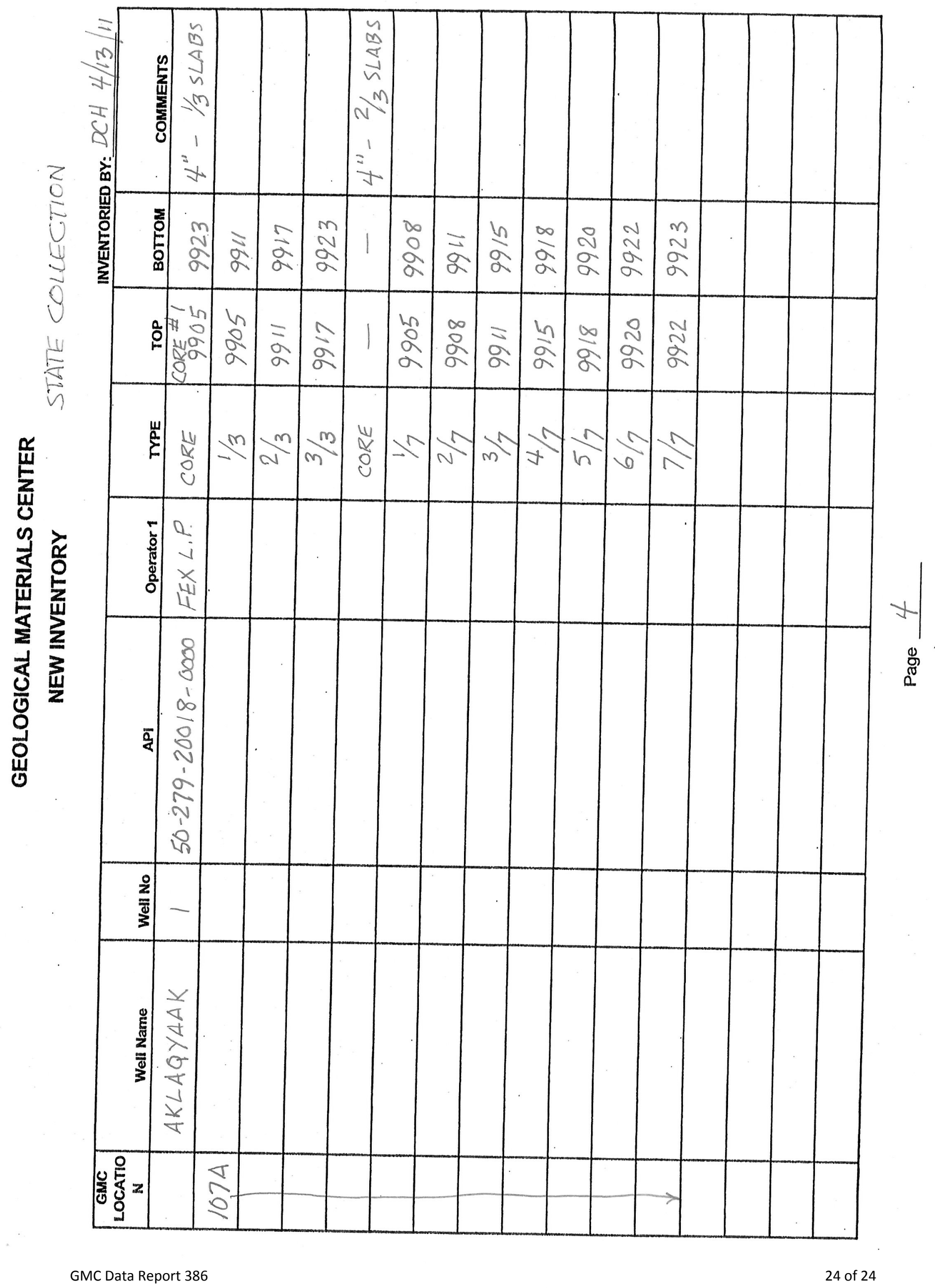

